\title{
Existence of periodic solutions for a class of $p$-Laplacian equations
}

\section{Xiaojun Chang ${ }^{1,2^{*}}$ and Yu Qiao ${ }^{3}$}

\author{
"Correspondence: \\ changxj1982@hotmail.com \\ 'School of Mathematics and \\ Statistics, Northeast Normal \\ University, Changchun, Jilin 130024, \\ P.R. China \\ ${ }^{2}$ College of Mathematics, Jilin \\ University, Changchun, Jilin 130012, \\ P.R. China \\ Full list of author information is \\ available at the end of the article
}

\begin{abstract}
This paper is devoted to the existence of periodic solutions for the one-dimensional p-Laplacian equation

$$
-\left(\phi_{p}\left(u^{\prime}\right)\right)^{\prime}=f(t, u)
$$

where $\phi_{p}(u)=|u|^{p-2} u(1<p<+\infty), f \in C([0,2 \pi] \times \mathbb{R}, \mathbb{R})$. By using some asymptotic interaction of the ratios $\frac{f(t, u)}{|u|^{p-2} u}$ and $\frac{p \int_{0}^{u} f(t, s) d s}{|u|^{p}}$ with the Fučík spectrum of $-\left(\phi_{p}\left(u^{\prime}\right)\right)^{\prime}$ related to periodic boundary condition, we establish a new existence theorem of periodic solutions for the one-dimensional $p$-Laplacian equation.
\end{abstract}

Keywords: periodic solutions; p-Laplacian; Fučík spectrum; Leray-Schauder degree; Borsuk theorem

\section{Introduction and main results}

In this paper, we are concerned with the existence of solutions for the following periodic boundary value problem:

$$
\left\{\begin{array}{l}
-\left(\phi_{p}\left(u^{\prime}\right)\right)^{\prime}=f(t, u), \\
u(0)=u(2 \pi), \quad u^{\prime}(0)=u^{\prime}(2 \pi)
\end{array}\right.
$$

where $\phi_{p}(u)=|u|^{p-2} u(1<p<+\infty), f \in C([0,2 \pi] \times \mathbb{R}, \mathbb{R})$. A solution $u$ of problem (1.1) means that $u$ is $C^{1}$ and $\phi_{p}\left(u^{\prime}\right)$ is absolutely continuous such that (1.1) is satisfied for a.e. $t \in[0,2 \pi]$.

Existence and multiplicity of solutions of the periodic problems driven by the $p$ Laplacian have been obtained in the literature by many people (see [1-5]). Many solvability conditions for problem (1.1) were established by using the asymptotic interaction at infinity of the ratio $\frac{f(x, u)}{|u|^{p-2} u}$ with the Fuč́lk spectrum for $\left(\phi_{p}\left(u^{\prime}\right)\right)^{\prime}$ under periodic boundary condition (see e.g., [2, 4, 6-9]). In [6], Del Pino, Manásevich and Murúa firstly defined the Fučík spectrum for $\left(\phi_{p}\left(u^{\prime}\right)\right)^{\prime}$ under periodic boundary value condition as the set $\Sigma_{p}$ consisting of all the pairs $\left(\lambda_{+}, \lambda_{-}\right) \in \mathbb{R}^{2}$ such that the equation

$$
-\left(\phi_{p}\left(u^{\prime}\right)\right)^{\prime}=\lambda_{+}\left(u^{+}\right)^{p-1}-\lambda_{-}\left(u^{-}\right)^{p-1}
$$

\section{Springer}

(c) 2013 Chang and Qiao; licensee Springer. This is an Open Access article distributed under the terms of the Creative Commons Attribution License (http://creativecommons.org/licenses/by/2.0), which permits unrestricted use, distribution, and reproduction in any medium, provided the original work is properly cited. 
admits at least one nontrivial $2 \pi$-periodic solution (see [10] for $p=2$ ). Let

$$
\pi_{p}=2(p-1)^{\frac{1}{p}} \int_{0}^{1}\left(1-t^{p}\right)^{-\frac{1}{p}} d t=2(p-1)^{\frac{1}{p}} \frac{\pi}{p \sin \left(\frac{\pi}{p}\right)} .
$$

By [6], it follows that

$$
\Sigma_{p}=\left\{\left(\lambda_{+}, \lambda_{-}\right) \in \mathbb{R}^{2}: \pi_{p}\left(\frac{1}{\sqrt[p]{\lambda_{+}}}+\frac{1}{\sqrt[p]{\lambda_{-}}}\right)=\frac{2 \pi}{k}, k \in \mathbb{Z}^{+}\right\} .
$$

Then they applied the Sturm's comparison theorem and Leray-Schauder degree theory to prove that problem (1.1) is solvable if the following relations hold:

$$
\begin{aligned}
& p_{1} \leq \liminf _{u \rightarrow+\infty} \frac{f(t, u)}{|u|^{p-2} u} \leq \limsup _{u \rightarrow+\infty} \frac{f(t, u)}{|u|^{p-2} u} \leq p_{2}, \\
& q_{1} \leq \liminf _{u \rightarrow-\infty} \frac{f(t, u)}{|u|^{p-2} u} \leq \limsup _{u \rightarrow-\infty} \frac{f(t, u)}{|u|^{p-2} u} \leq q_{2},
\end{aligned}
$$

uniformly for a.e. $t \in[0,2 \pi]$ with $p_{1}, q_{1}, p_{2}, q_{2}>0$ satisfying

$$
\frac{2 \pi}{(k+1) \pi_{p}}<\frac{1}{\sqrt[p]{p_{2}}}+\frac{1}{\sqrt[p]{q_{2}}} \leq \frac{1}{\sqrt[p]{p_{1}}}+\frac{1}{\sqrt[p]{q_{1}}}<\frac{2 \pi}{k \pi_{p}}, \quad k \in \mathbb{Z}^{+} .
$$

Clearly, in this case, we have $\left(\left[p_{1}, q_{1}\right] \times\left[p_{2}, q_{2}\right]\right) \cap \Sigma_{p}=\emptyset$, which is usually called that the nonlinearity $f$ is nonresonant with respect to the Fućík spectrum $\Sigma_{p}$. In [11], Anane and Dakkak obtained a similar result by using the property of nodal set for eigenfunctions. If $f$ is resonant with respect to $\Sigma_{p}$, i.e., there exists $\left(\lambda_{+}, \lambda_{-}\right) \in \Sigma_{p}$ such that $\lim _{u \rightarrow+\infty} \frac{f(t, u)}{|u|^{p-2} u}=\lambda_{+}$, $\lim _{u \rightarrow-\infty} \frac{f(t, u)}{|u|^{-2} u}=\lambda_{-}$uniformly for a.e. $t \in[0,2 \pi]$, together with the Landesman-Lazer type condition, Jiang [9] obtained the existence of solutions of (1.1) by applying the variational methods and symplectic transformations. In these works, either $f$ is resonant or nonresonant with respect to $\Sigma_{p}$, the solvability of problem (1.1) was assured by assuming that the ratio $\frac{f(t, u)}{|u|^{-2} u}$ stays at infinity in the pointwise sense asymptotically between two consecutive curves of $\Sigma_{p}$. Note that

$$
\liminf _{s \rightarrow \pm \infty} \frac{f(t, s)}{s} \leq \liminf _{s \rightarrow \pm \infty} \frac{2 F(t, s)}{s^{2}} \leq \limsup _{s \rightarrow \pm \infty} \frac{2 F(t, s)}{s^{2}} \leq \limsup _{s \rightarrow \pm \infty} \frac{f(t, s)}{s}
$$

we can see that the conditions on the ratio $\frac{2 F(t, s)}{s^{2}}$ are more general than that on the ratio $\frac{f(t, s)}{s}$. Recently, Liu and Li [2] studied the nondissipative $p$-Laplacian equation

$$
-\left(\phi_{p}\left(u^{\prime}\right)\right)^{\prime}=c\left(\phi_{p}\left(u^{\prime}\right)\right)^{\prime}+g(u)-p(t)
$$

where $c>0$ is a constant. Define $G(u)=\int_{0}^{u} g(s) d s$. They proved that (1.2) is solvable under the following assumptions:

(1) There exist $b, d_{1}, d_{2}>0$ such that $d_{1} \leq \frac{g(u)}{|u|^{-2} u} \leq d_{2}$ for all $|u| \geq b$;

(2) $\lim _{u \rightarrow+\infty} \frac{p G(u)}{|u|^{p}}=\lambda_{+}, \lim _{u \rightarrow-\infty} \frac{p G(u)}{|u|^{p}}=\lambda_{-}$with $\left(\lambda_{+}, \lambda_{-}\right) \notin \Sigma_{p}$.

Here, the potential function $G$ is nonresonant with respect to $\Sigma_{p}$ and the ratio $\frac{g(u)}{|u|^{p-2} u}$ is not required to stay at infinity in the pointwise sense asymptotically between two consecutive branches of $\Sigma_{p}$ and it may even cross at infinity multiple Fućík spectrum curves. 
In this paper, we want to obtain the solvability of problem (1.1) by using the asymptotic interaction at infinity of both the ratios $\frac{f(x, u)}{|u|^{-2} u}$ and $\frac{p F(t, u)}{|u|^{p}}$ with the Fućík spectrum for $\left(\phi_{p}\left(u^{\prime}\right)\right)^{\prime}$ under periodic boundary condition. Here, $F(t, u)=\int_{0}^{u} f(t, s) d s$. The goal is to obtain the existence of solutions of (1.1) by requiring neither the ratio $\frac{f(x, u)}{|u|^{p-2} u}$ stays at infinity in the pointwise sense asymptotically between two consecutive branches of $\Sigma_{p}$ nor the $\operatorname{limits}_{\lim _{u \rightarrow \pm \infty}} \frac{p F(t, u)}{|u|^{p}}$ exist. We shall prove that problem (1.1) admits a solution under the assumptions that the nonlinearity $f$ has at most $(p-1)$-linear growth at infinity and the ratio $\frac{f(t, u)}{|u|^{-2} u}$ has a $L^{1}$ limit as $u \rightarrow \pm \infty$, while the ratio $\frac{p F(t, u)}{|u|^{p}}$ stays at infinity in the pointwise sense asymptotically between two consecutive branches of $\Sigma_{p}$. Our result will complement the results in the literature on the solvability of problem (1.1) involving the Fuč́k spectrum.

For related works on resonant problems involving the Fučík spectrum, we also refer the interested readers to see [12-19] and the references therein.

Our main result for problem (1.1) now reads as follows.

Theorem 1.1 Assume that $f \in C([0,2 \pi] \times \mathbb{R})$ and the following conditions hold:

(i) There exist constants $C_{1}, M>0$ such that

$$
|f(t, u)| \leq C_{1}\left(1+|u|^{p-1}\right), \quad \text { a.e. } t \in[0,2 \pi], \forall|u| \geq M ;
$$

(ii) There exists $\eta^{ \pm} \in L^{\infty}(0,2 \pi)$ such that

$$
\int_{0}^{2 \pi}\left|\frac{f(t, u)}{|u|^{p-2} u}-\eta^{ \pm}(t)\right| d t \rightarrow 0 \quad \text { as } u \rightarrow \pm \infty
$$

(iii) There exist constants $p_{1}, p_{2}, q_{1}, q_{2}>0$ such that

$$
\begin{aligned}
& p_{1} \leq \liminf _{u \rightarrow+\infty} \frac{p F(t, u)}{|u|^{p}} \leq \limsup _{u \rightarrow+\infty} \frac{p F(t, u)}{|u|^{p}} \leq p_{2}, \\
& q_{1} \leq \liminf _{u \rightarrow-\infty} \frac{p F(t, u)}{|u|^{p}} \leq \limsup _{u \rightarrow-\infty} \frac{p F(t, u)}{|u|^{p}} \leq q_{2}
\end{aligned}
$$

hold uniformly for a.e. $t \in[0,2 \pi]$ with

$$
\left(\left[p_{1}, p_{2}\right] \times\left[q_{1}, q_{2}\right]\right) \cap \Sigma_{p}=\emptyset .
$$

Then problem (1.1) admits a solution.

Remark If $f(t, u)=a(t)|u|^{p-2} u^{+}-b(t)|u|^{p-2} u^{-}+e(u)+h(t)$, where $a, b, h \in C[0,2 \pi]$ with $p_{1} \leq a(t) \leq p_{2}, q_{1} \leq b(t) \leq q_{2}$ and $p_{1}, p_{2}, q_{1}, q_{2}>0$ satisfy (1.7), $e$ is continuous on $\mathbb{R}$ and $\lim _{u \rightarrow+\infty} \frac{e(u)}{|u|^{-2} u}=0$, then $\lim _{u \rightarrow+\infty} \frac{p F(t, u)}{|u|^{p}}=a(t)$ and $\lim _{u \rightarrow-\infty} \frac{p F(t, u)}{|u|^{p}}=b(t)$. By Theorem 1.1, it follows that problem (1.1) admits a solution. It is easily seen that the result of [16] cannot be applied to this case. Note that one can also obtain the solvability of (1.1) in this case by the result of [6], while in Theorem 1.1 we do not require the pointwise limit at infinity of the ratio $\frac{f(t, u)}{|u|^{p-2} u}$ as in [6].

For convenience, we introduce some notations and definitions. $L^{p}(0,2 \pi)(1<p<\infty)$ denotes the usual Sobolev space with inner product $\langle\cdot, \cdot\rangle_{p}$ and norm $\|\cdot\|_{p}$, respectively. 
$C^{m}[0,2 \pi](m \in \mathbb{N})$ denotes the space of $m$-times continuous differential real functions with norm

$$
\|x\|_{C^{m}}=\max _{t \in[0,2 \pi]}|x(t)|+\max _{t \in[0,2 \pi]}|\dot{x}(t)|+\cdots+\max _{t \in[0,2 \pi]}\left|x^{(m)}(t)\right| .
$$

\section{Proof of the main result}

Denote by deg the Leray-Schauder degree. To prove Theorem 1.1, we need the following results.

Lemma 2.1 [20] Let $\Omega$ be a bounded open region in a real Banach space X. Assume that $K: \bar{\Omega} \rightarrow \mathbb{R}$ is completely continuous and $p \notin(I-K)(\partial \Omega)$. Then the equation $(I-K)(x)=p$ has a solution in $\Omega$ if $\operatorname{deg}(I-K, \Omega, p) \neq 0$.

Lemma 2.2 (Borsuk Theorem [20]) Assume that $X$ is a real Banach space. Let $\Omega$ be a symmetric bounded open region with $\theta \in \Omega$. Assume that $K: \bar{\Omega} \rightarrow \mathbb{R}$ is completely continuous and odd with $\theta \notin(I-K)(\partial \Omega)$. Then $\operatorname{deg}(I-K, \Omega, \theta)$ is odd .

Proof of Theorem 1.1 Take $\left(\lambda_{+}, \lambda_{-}\right) \in\left[p_{1}, p_{2}\right] \times\left[q_{1}, q_{2}\right]$. Consider the following homotopy problem:

$$
\left\{\begin{array}{l}
-\left(\phi_{p}\left(u^{\prime}\right)\right)^{\prime}=(1-\mu)\left(\lambda_{+}\left(u^{+}\right)^{p-1}-\lambda_{-}\left(u^{-}\right)^{p-1}\right)+\mu f(t, u) \equiv f_{\mu}(t, u) \\
u(0)=u(2 \pi), \quad u^{\prime}(0)=u^{\prime}(2 \pi)
\end{array}\right.
$$

where $\mu \in[0,1]$.

By (1.3) and the regularity arguments, it follows that $u \in C^{1}[0,2 \pi]$, and furthermore there exists $a, b \in \mathbb{R}^{+}$such that, if $u$ is a solution of problem (2.1), then

$$
\|u\|_{C^{1}} \leq a\|u\|_{\infty}+b
$$

In what follows, we shall prove that there exists $C>0$ independent of $\mu \in[0,1]$ such that $\|u\|_{\infty} \leq C$ for all possible solution $u(t)$ of (2.1). Assume by contradiction that there exist a sequence of number $\left\{\mu_{n}\right\} \subset[0,1]$ and corresponding solutions $\left\{u_{n}\right\}$ of $(2.1)$ such that

$$
\left\|u_{n}\right\|_{\infty} \rightarrow+\infty \quad \text { as } n \rightarrow+\infty
$$

Set $z_{n}=\frac{u_{n}}{\left\|u_{n}\right\|_{\infty}}$. Obviously, $\left\|z_{n}\right\|_{\infty}=1$. Define

$$
\alpha_{n}(t)= \begin{cases}\frac{f\left(t, u_{n}\right)}{\left|u_{n}\right|^{p-2} u_{n}}, & u_{n}(t)>M \\ 0, & u_{n}(t) \leq M\end{cases}
$$

and

$$
\beta_{n}(t)= \begin{cases}\frac{f\left(t, u_{n}\right)}{\left|u_{n}\right|^{p-2} u_{n}}, & u_{n}(t)<-M \\ 0, & u_{n}(t) \geq-M\end{cases}
$$


By (1.3), there exists $M_{0}>0$ such that

$$
\left|\alpha_{n}(t)\right|,\left|\beta_{n}(t)\right| \leq M_{0}, \quad \text { a.e. } t \in[0,2 \pi]
$$

Then there exist $\alpha_{0}, \beta_{0} \in L^{\infty}(0,2 \pi)$ such that

$$
\alpha_{n}(t) \stackrel{*}{\rightarrow} \alpha_{0}(t), \quad \beta_{n}(t) \stackrel{*}{\rightarrow} \beta_{0}(t) \quad \text { in } L^{\infty}(0,2 \pi) .
$$

In addition, using (1.3) and the regularity arguments, there exists $M_{1}>0$ such that, for each $n$, we have $\left\|z_{n}\right\|_{C^{1}} \leq M_{1}$, and thus there exists $z_{0} \in C^{1}[0,2 \pi]$ such that, passing to a subsequence if possible,

$$
z_{n} \rightarrow z_{0} \quad \text { in } C^{1}[0,2 \pi]
$$

Clearly, $\left\|z_{0}\right\|_{\infty}=1$. In view of $\left\{\mu_{n}\right\} \subset[0,1]$, there exists $\mu_{0} \in[0,1]$ such that, passing to a subsequence if possible,

$$
\mu_{n} \rightarrow \mu_{0} \quad \text { as } n \rightarrow+\infty
$$

Note that for $\mu=0$, problem (2.1) has only the trivial solution, it follows that $\mu_{0} \in(0,1]$. Denote $\bar{\alpha}(t)=\left(1-\mu_{0}\right) \lambda_{+}+\mu_{0} \alpha_{0}(t), \bar{\beta}(t)=\left(1-\mu_{0}\right) \lambda_{-}+\mu_{0} \beta_{0}(t)$. It is easily seen that $z_{0}$ is a nontrivial solution of the following problem:

$$
\left\{\begin{array}{l}
-\left(\phi_{p}\left(z_{0}^{\prime}\right)\right)^{\prime}=\bar{\alpha}(t)\left(z_{0}^{+}\right)^{p-1}-\bar{\beta}(t)\left(z_{0}^{-}\right)^{p-1}, \\
z_{0}(0)=z_{0}(2 \pi), \quad z_{0}^{\prime}(0)=z_{0}^{\prime}(2 \pi) .
\end{array}\right.
$$

We now distinguish three cases:

(i) $z_{0}$ changes sign in $[0,2 \pi]$;

(ii) $z_{0}(t) \geq 0, \forall t \in[0,2 \pi]$;

(iii) $z_{0}(t) \leq 0, \forall t \in[0,2 \pi]$.

In the following, it will be shown that each case leads to a contradiction.

Case (i). Let

$$
\begin{aligned}
& I^{+}=\left\{t \in[0,2 \pi]: z_{0}(t)>0\right\}, \\
& I^{-}=\left\{t \in[0,2 \pi]: z_{0}(t)<0\right\}, \\
& I^{0}=\left\{t \in[0,2 \pi]: z_{0}(t)=0\right\} .
\end{aligned}
$$

Then, as $n \rightarrow+\infty$, we get

$$
\begin{aligned}
& u_{n}(t) \rightarrow+\infty, \quad \forall t \in I^{+}, \\
& u_{n}(t) \rightarrow-\infty, \quad \forall t \in I^{-} .
\end{aligned}
$$

In addition, as shown in [11], we have $\left|I^{0}\right|=0$. Define

$$
\eta_{+}(t)= \begin{cases}\eta(t), & t \in I^{+}, \\ \alpha_{0}(t), & t \in I^{-}\end{cases}
$$


and

$$
\eta_{-}(t)= \begin{cases}\beta_{0}(t), & t \in I^{+} \\ \eta(t), & t \in I^{-}\end{cases}
$$

By (1.4) and (2.4), it follows that

$$
\alpha_{0}(t) \equiv \eta_{+}(t), \quad \beta_{0}(t) \equiv \eta_{-}(t), \quad \text { a.e. } t \in[0,2 \pi] .
$$

Thus, $z_{0}$ satisfies

$$
\left\{\begin{array}{l}
-\left(\phi_{p}\left(z_{0}^{\prime}\right)\right)^{\prime}=\tilde{\alpha}(t)\left(z_{0}^{+}\right)^{p-1}-\tilde{\beta}(t)\left(z_{0}^{-}\right)^{p-1} \\
z_{0}(0)=z_{0}(2 \pi), \quad z_{0}^{\prime}(0)=z_{0}^{\prime}(2 \pi)
\end{array}\right.
$$

Here, $\tilde{\alpha}(t)=\left(1-\mu_{0}\right) \lambda_{+}+\mu_{0} \eta_{+}(t), \tilde{\beta}(t)=\left(1-\mu_{0}\right) \lambda_{-}+\mu_{0} \eta_{-}(t)$.

Now we prove that there exist $\bar{n} \in \mathbb{Z}^{+}$and $0<\kappa_{1}<1<\kappa_{2}$ such that

$$
\kappa_{1} \leq \frac{\max u_{n}}{-\min u_{n}} \leq \kappa_{2}, \quad \forall n \geq \bar{n}
$$

In fact, if not, we assume, by contradiction, that there exists a subsequence of $\left\{u_{n}\right\}$, we still denote it as $\left\{u_{n}\right\}$ with $\max u_{n} \rightarrow \infty$ and $\min u_{n} \rightarrow-\infty$, such that

$$
\frac{\max u_{n}}{-\min u_{n}} \rightarrow 0 \quad \text { or } \quad \frac{\max u_{n}}{-\min u_{n}} \rightarrow+\infty
$$

Combing with (2.5), $\left\|z_{0}\right\|_{\infty}=1$ and the fact that $z_{0}$ changes sign, we obtain

$$
\max \frac{u_{n}}{\left\|u_{n}\right\|_{\infty}} /\left(-\min \frac{u_{n}}{\left\|u_{n}\right\|_{\infty}}\right) \rightarrow \frac{\max z_{0}}{-\min z_{0}}>0
$$

A contradiction. Hence, (2.9) holds.

For any $(t, \mu) \in[0,2 \pi] \times[0,1]$, define

$$
\begin{aligned}
& \bar{f}_{1}(t, s, \mu)=f_{\mu}(t, s)-\tilde{\alpha}(t) s^{p-1}, \quad \forall s \in \mathbb{R}^{+}, \\
& \bar{f}_{2}(t, r, \mu)=f_{\mu}(t, r)-\tilde{\beta}(t)|r|^{p-2} r, \quad \forall r \in \mathbb{R}^{-}
\end{aligned}
$$

and

$$
\bar{F}_{1}(t, s, \mu)=\int_{0}^{s} \bar{f}_{1}(t, \tau, \mu) d \tau, \quad \bar{F}_{2}(t, r, \mu)=\int_{0}^{r} \bar{f}_{2}(t, \tau, \mu) d \tau .
$$

Denote $s_{n}=\max u_{n}, r_{n}=\min u_{n}$. Then by (2.9) it follows that $s_{n} \rightarrow+\infty$ and $r_{n} \rightarrow-\infty$. Taking $t_{n}$ such that $u_{n}\left(t_{n}\right)=s_{n}, t_{n}^{0}$ is the nearest point satisfying $t_{n}^{0}<t_{n}$ and $u_{n}\left(t_{n}^{0}\right)=0$. Since $t_{n}^{0}, t_{n} \in[0,2 \pi]$, there exist $\bar{t}^{0}, \bar{t} \in[0,2 \pi]$ such that

$$
t_{n}^{0} \rightarrow \bar{t}^{0}, \quad t_{n} \rightarrow \bar{t} \quad \text { as } n \rightarrow+\infty
$$


By (2.5), we obtain $z_{0}\left(\bar{t}^{0}\right)=0, z_{0}(\bar{t})=\max _{t \in[0,2 \pi]} z_{0}(t)$. Note that $\left\|u_{n}\right\|_{\infty} \rightarrow+\infty$, we have $u_{n}(t) \rightarrow+\infty, \forall t \in\left(\bar{t}^{0}, \bar{t}\right)$. Hence, together with $\mu_{n} \rightarrow \mu_{0}$ and (1.4), there exist subsequences of $\left\{u_{n}\right\}$ and $\left\{\mu_{n}\right\}$, we still denote them as $\left\{u_{n}\right\}$ and $\left\{\mu_{n}\right\}$, such that, for a.e. $t \in[0,2 \pi]$,

$$
\frac{\bar{f}_{1}\left(t, u_{n}(\tau), \mu_{n}\right)}{\left(u_{n}(\tau)\right)^{p-1}} \rightarrow 0, \quad \text { a.e. } \tau \in\left(\bar{t}^{0}, \bar{t}\right)
$$

Using (1.3), for a.e. $t \in[0,2 \pi],\left\{\frac{\bar{f}_{1}\left(t, u_{n}(\tau), \mu_{n}\right)}{\left(u_{n}(\tau)\right)^{p-1}}\right\}$ is uniformly bounded with respect to $\tau \in\left(\bar{t}^{0}, \bar{t}\right)$, we obtain by the Lebesgue dominated convergence theorem that

$$
\int_{\bar{t}^{0}}^{\bar{t}}\left|\frac{\bar{f}_{1}\left(t, u_{n}(\tau), \mu_{n}\right)}{\left(u_{n}(\tau)\right)^{p-1}}\right| d \tau \rightarrow 0, \quad \text { uniformly for a.e. } t \in[0,2 \pi]
$$

Thus,

$$
\begin{aligned}
& \int_{t_{n}^{0}}^{t_{n}}\left|\frac{\bar{f}_{1}\left(t, u_{n}(\tau), \mu_{n}\right)}{\left(u_{n}(\tau)\right)^{p-1}}\right| d \tau \\
& \quad=\int_{t_{n}^{0}}^{\bar{t}^{0}}\left|\frac{\bar{f}_{1}\left(t, u_{n}(\tau), \mu_{n}\right)}{\left(u_{n}(\tau)\right)^{p-1}}\right| d \tau+\int_{\bar{t}^{0}}^{\bar{t}}\left|\frac{\bar{f}_{1}\left(t, u_{n}(\tau), \mu_{n}\right)}{\left(u_{n}(\tau)\right)^{p-1}}\right| d \tau+\int_{\bar{t}}^{t_{n}}\left|\frac{\bar{f}_{1}\left(t, u_{n}(\tau), \mu_{n}\right)}{\left(u_{n}(\tau)\right)^{p-1}}\right| d t \\
& \quad \rightarrow 0, \quad \text { uniformly for a.e. } t \in[0,2 \pi] .
\end{aligned}
$$

By (1.4) and (2.2), we get

$$
\begin{aligned}
& \left|p \bar{F}_{1}\left(t, s_{n}, \mu_{n}\right)\right| \\
& =\left|p \bar{F}_{1}\left(t, u_{n}\left(t_{n}\right), \mu_{n}\right)-p \bar{F}_{1}\left(t, u_{n}\left(t_{n}^{0}\right), \mu_{n}\right)\right| \\
& \quad=\left|\int_{t_{n}^{0}}^{t_{n}} p \bar{f}_{1}\left(t, u_{n}(\tau), \mu_{n}\right) u_{n}^{\prime}(\tau) d \tau\right| \\
& \quad \leq p \int_{t_{n}^{0}}^{t_{n}}\left|\bar{f}_{1}\left(t, u_{n}(\tau), \mu_{n}\right)\right| d \tau\left\|u_{n}^{\prime}\right\|_{\infty} \\
& \quad=p \int_{t_{n}^{0}}^{t_{n}}\left|\frac{\bar{f}_{1}\left(t, u_{n}(\tau), \mu_{n}\right)}{\left(u_{n}(\tau)\right)^{p-1}}\right|\left|\left(z_{n}(\tau)\right)^{p-1}\right| d \tau\left\|u_{n}\right\|_{\infty}^{p-1}\left\|u_{n}^{\prime}\right\|_{\infty} \\
& \quad \leq C \int_{t_{n}^{0}}^{t_{n}}\left|\frac{\bar{f}_{1}\left(t, u_{n}(\tau), \mu_{n}\right)}{\left(u_{n}(\tau)\right)^{p-1}}\right| d \tau s_{n}^{p} .
\end{aligned}
$$

In view of (2.11), we obtain that

$$
\left|\frac{p \bar{F}_{1}\left(t, s_{n}, \mu_{n}\right)}{s_{n}^{p}}\right| \rightarrow 0
$$

holds uniformly for a.e. $t \in[0,2 \pi]$. Similarly,

$$
\left|\frac{p \bar{F}_{2}\left(t, r_{n}, \mu_{n}\right)}{\left|r_{n}\right|^{p}}\right| \rightarrow 0
$$

holds uniformly for a.e. $t \in[0,2 \pi]$. 
On the other hand, for $\left\{s_{n}\right\},\left\{r_{n}\right\}$ satisfying (2.12)-(2.13), denoting

$$
\begin{aligned}
& \xi_{1}(t)=\liminf _{n \rightarrow+\infty} \frac{p F\left(t, s_{n}\right)}{\left|s_{n}\right|^{p}}, \\
& \xi_{2}(t)=\liminf _{n \rightarrow+\infty} \frac{p F\left(t, r_{n}\right)}{\left|r_{n}\right|^{p}},
\end{aligned}
$$

we obtain by (1.5)-(1.6) that

$$
p_{1} \leq \xi_{1}(t) \leq q_{1}, \quad p_{2} \leq \xi_{2}(t) \leq q_{2}, \quad \text { a.e. } t \in[0,2 \pi] .
$$

Using $\mu_{n} \rightarrow \mu_{0}$, we have

$$
\begin{aligned}
& \liminf _{n \rightarrow+\infty} \frac{p \bar{F}_{1}\left(t, s_{n}, \mu_{n}\right)}{\left|s_{n}\right|^{p}} \\
& \quad=\liminf _{n \rightarrow+\infty}\left[\left(1-\mu_{n}\right) \lambda_{+}+\mu_{n} \frac{p F\left(t, s_{n}\right)}{\left|s_{n}\right|^{p}}-\tilde{\alpha}(t)\right] \\
& \quad=\mu_{0}\left(\xi_{1}(t)-\eta_{+}(t)\right), \quad \text { a.e. } t \in[0,2 \pi], \\
& \liminf _{n \rightarrow+\infty} \frac{p \bar{F}_{2}\left(t, r_{n}, \mu_{n}\right)}{\left|r_{n}\right|^{p}} \\
& \quad=\liminf _{n \rightarrow+\infty}\left[\left(1-\mu_{n}\right) \lambda_{-}+\mu_{n} \frac{p F\left(t, r_{n}\right)}{\left|r_{n}\right|^{p}}-\tilde{\beta}(t)\right] \\
& =\mu_{0}\left(\xi_{2}(t)-\eta_{-}(t)\right), \quad \text { a.e. } t \in[0,2 \pi] .
\end{aligned}
$$

We claim that there exists subinterval $I_{1} \subset[0,2 \pi]$ with $\left|I_{1}\right|>0$ such that

$$
\xi_{1}(t)-\eta_{+}(t) \neq 0, \quad \forall t \in I_{1},
$$

or subinterval $I_{2} \subset[0,2 \pi]$ with $\left|I_{2}\right|>0$ such that

$$
\xi_{2}(t)-\eta_{-}(t) \neq 0, \quad \forall t \in I_{2} .
$$

Indeed, if not, we assume that $\eta_{+}(t)=\xi_{1}(t), \eta_{-}(t)=\xi_{2}(t)$, a.e. $t \in[0,2 \pi]$. Together with the choosing of $\lambda_{+}, \lambda_{-}$and (2.14), we get

$$
p_{1} \leq \tilde{\alpha}(t) \leq q_{1}, \quad p_{2} \leq \tilde{\beta}(t) \leq q_{2}, \quad \text { a.e. } t \in[0,2 \pi] .
$$

Then by (1.7), it follows that $z_{0} \equiv 0$. A contradiction. Combining (2.12)-(2.13) with (2.15)(2.18), we obtain a contradiction.

Case (ii). In this case, we have

$$
s_{n} \rightarrow+\infty \text { and }\left\{r_{n}\right\} \text { is uniformly bounded. }
$$

Using similar arguments as in Case (i), by (1.4) and (2.4) it follows that $\alpha_{0}(t) \equiv \eta_{+}(t), \forall t \in$ $[0,2 \pi]$. Taking $\bar{f}^{+}=\bar{f}_{1}, \bar{F}^{+}=\bar{F}_{1}$, a.e. $t \in[0,2 \pi]$. We can see that there exists subsequence 
of $\left\{s_{n}\right\}$, which is still denoted by $\left\{s_{n}\right\}$, such that

$$
\left|\frac{p \bar{F}^{+}\left(t, s_{n}, \mu_{n}\right)}{s_{n}^{p}}\right| \rightarrow 0
$$

holds uniformly for a.e. $t \in[0,2 \pi]$. On the other hand, for $\left\{s_{n}\right\}$ satisfying (2.19), denoting

$$
\xi^{+}(t)=\liminf _{n \rightarrow+\infty} \frac{p F\left(t, s_{n}\right)}{\left|s_{n}\right|^{p}}
$$

we obtain by (1.5) that

$$
p_{1} \leq \xi^{+}(t) \leq q_{1}, \quad \text { a.e. } t \in[0,2 \pi]
$$

Using $\mu_{n} \rightarrow \mu_{0}$, we have

$$
\begin{aligned}
& \liminf _{n \rightarrow+\infty} \frac{p \bar{F}^{+}\left(t, s_{n}, \mu_{n}\right)}{\left|s_{n}\right|^{p}} \\
& \quad=\liminf _{n \rightarrow+\infty}\left[\left(1-\mu_{n}\right) \lambda_{+}+\mu_{n} \frac{p F\left(s_{n}\right)}{\left|s_{n}\right|^{p}}-\tilde{\alpha}(t)\right] \\
& \quad=\mu_{0}\left(\xi^{+}(t)-\eta_{+}(t)\right), \quad \text { a.e. } t \in[0,2 \pi] .
\end{aligned}
$$

We shall show that there exists subinterval $I_{+} \subset[0,2 \pi]$ with $\left|I_{+}\right|$such that

$$
\xi^{+}(t)-\eta_{+}(t) \neq 0, \quad \forall t \in I_{+} .
$$

In fact, if not, we assume that $\eta_{+}(t)=\xi^{+}(t)$, a.e. $t \in[0,2 \pi]$. By the choosing of $\lambda_{+}$and $(2.20)$, we get $p_{1} \leq \tilde{\alpha}(t) \leq q_{1}$, a.e. $t \in[0,2 \pi]$. Thus, $z_{0}$ is a nontrivial solution of the following problem:

$$
\left\{\begin{array}{l}
-\left(\phi_{p}\left(z_{0}^{\prime}\right)\right)^{\prime}=\tilde{\alpha}(t) z_{0}^{p-1}, \\
z_{0}(0)=z_{0}(2 \pi), \quad z_{0}^{\prime}(0)=z_{0}^{\prime}(2 \pi) .
\end{array}\right.
$$

Taking 1 as test function in problem (2.23), we get

$$
0=\int_{0}^{2 \pi} \tilde{\alpha}(t) z_{0}^{p-1} d t
$$

By $\tilde{\alpha}(t) \geq p_{1}>0$ for a.e. $t \in[0,2 \pi]$, it follows that $z_{0}(t)=0$ for a.e. $t \in[0,2 \pi]$, which is contrary to that $\left\|z_{0}\right\|_{\infty}=1$. Hence, (2.22) holds. Clearly, (2.21)-(2.22) contradict (2.19).

Case (iii). In this case, $r_{n} \rightarrow-\infty$ and $\left\{s_{n}\right\}$ is uniformly bounded. Similar arguments as in Case (ii) imply a contradiction.

In a word, (2.3) cannot hold, and hence by (2.2) there exists $C>0$ independent of $\mu \in$ $[0,1]$ such that, if $u$ is a solution of problem (2.1), then

$$
\|u\|_{C^{1}} \leq C
$$


Note that, for each $h \in L^{\infty}(0,2 \pi)$, the problem

$$
\left\{\begin{array}{l}
-\left(\phi_{p}\left(u^{\prime}\right)\right)^{\prime}+\phi_{p}(u)=h(t), \\
u(0)=u(2 \pi), \quad u^{\prime}(0)=u^{\prime}(2 \pi)
\end{array}\right.
$$

has a unique solution $G_{p}(h) \in C^{1}[0,2 \pi]$. Clearly, the operator $G_{p}$ seen as an operator from $C[0,2 \pi]$ into $C^{1}[0,2 \pi]$ is completely continuous. Define $\psi: C^{1}[0,2 \pi] \rightarrow C[0,2 \pi]$ by $\psi(u)(t)=f(t, u(t))$. Then solving problem (1.1) is equivalent to finding solutions in $C^{1}[0,2 \pi]$ of the equation

$$
u-G_{p}(\psi(u))=0 .
$$

Let $(\alpha, \beta) \in\left[p_{1}, q_{1}\right] \times\left[p_{1}, q_{2}\right]$. Define the operator $T_{\alpha, \beta}: C^{1}[0,2 \pi] \rightarrow C^{1}[0,2 \pi]$ by $T_{\alpha, \beta}(u)=$ $G_{p}\left(\phi_{p}(u)+\alpha\left(u^{+}\right)^{p-1}-\beta\left(u^{-}\right)^{p-1}\right)$. Denote $B_{R}=\left\{u \in C^{1}[0,2 \pi]:\|u\|_{C^{1}}<R, R \in \mathbb{R}\right\}$. Clearly, $\operatorname{deg}\left(I-T_{\alpha, \beta}, B_{R}, 0\right)$ is well defined for all $R>0$. Owing to $\lambda_{+} \cdot \lambda_{-}>0$, there is a continuous curve $\alpha(\tau), \beta(\tau), \tau \in[0,1]$, whose image is in $\mathbb{R}^{2} \backslash \Sigma_{p}$ and $(\lambda, \lambda) \in \mathbb{R} \backslash \Sigma_{p}$ such that $(\alpha(0), \beta(0))=\left(\lambda_{+}, \lambda_{-}\right),(\alpha(1), \beta(1))=(\lambda, \lambda)$. From the invariance property of Leray-Schauder degree under compact homotopies, it follows that the degree $\operatorname{deg}\left(I-T_{\alpha(\tau), \beta(\tau)}, B_{R}, 0\right)$ is constant for $\tau \in[0,1]$. Obviously, the operator $T_{\lambda, \lambda}$ is odd. By the Borsuk's theorem, it follows that $\operatorname{deg}\left(I-T_{\lambda, \lambda}, B_{R}, 0\right) \neq 0$ for all $R>0$. Thus,

$$
\operatorname{deg}\left(I-T_{\lambda_{+}, \lambda_{-}}, B_{R}, 0\right) \neq 0, \quad \forall R>0 .
$$

Consider the following homotopy:

$$
H(\mu, u)=G_{p}\left(\phi_{p}(u)+(1-\mu)\left(\lambda_{+}\left(u^{+}\right)^{p-1}-\lambda_{-}\left(u^{-}\right)^{p-1}\right)+\mu \psi(u)\right)
$$

for $(\mu, u) \in[0,1] \times C^{1}[0,2 \pi]$. By $(2.25)$, we can see that there exists $R_{0}>0$ such that

$$
H(\mu, u) \neq u, \quad \forall \mu \in[0,1], \forall u \in \partial B_{R_{0}} .
$$

From the invariance property of Leray-Schauder degree, it follows that

$$
\begin{aligned}
\operatorname{deg}\left(I-H(1, \cdot), B_{R_{0}}, 0\right) & =\operatorname{deg}\left(I-H(0, \cdot), B_{R_{0}}, 0\right) \\
& =\operatorname{deg}\left(I-T_{\lambda_{+}, \lambda_{-}}, B_{R_{0}}, 0\right) \neq 0 .
\end{aligned}
$$

Hence, problem (1.1) has a solution. The proof is complete.

\section{Competing interests \\ The authors declare that they have no competing interests.}

Authors' contributions

All authors read and approved the final manuscript.

\section{Author details}

${ }^{1}$ School of Mathematics and Statistics, Northeast Normal University, Changchun, Jilin 130024, P.R. China. ${ }^{2}$ College of Mathematics, Jilin University, Changchun, Jilin 130012, P.R. China. ${ }^{3}$ College of Mathematics and Information Science, Shaanxi Normal University, Xi'an, 710062, P.R. China. 


\section{Acknowledgements}

The first author sincerely thanks Professor Yong Li and Doctor Yixian Gao for their many useful suggestions and the both authors thank Professor Zhi-Qiang Wang for many helpful discussions and his invitation to Chern Institute of Mathematics. The first author is partially supported by the NSFC Grant (11101178), NSFJP Grant (201215184) and FSIIP of Jilin University (201103203). The second author is partially sup- ported by NSFC Grant (11226123).

Received: 26 September 2012 Accepted: 5 April 2013 Published: 19 April 2013

\section{References}

1. Aizicovici, S, Papageorgiou, NS, Staicu, V: Nonlinear resonant periodic problems with concave terms. J. Math. Anal. Appl. 375, 342-364 (2011)

2. Liu, W, Li, Y: Existence of periodic solutions for $p$-Laplacian equation under the frame of Fučík spectrum. Acta Math. Sin. Engl. Ser. 27, 545-554 (2011)

3. Manásevich, R, Mawhin, J: Periodic solutions for nonlinear systems with $p$-Laplacian-like operators. J. Differ. Equ. 145 367-393 (1998)

4. Reichel, W, Walter, W: Sturm-Liouville type problems for the $p$-Laplacian under asymptotic nonresonance conditions. J. Differ. Equ. 156, 50-70 (1999)

5. Yang, X, Kim, Y, Lo, K: Periodic solutions for a generalized $p$-Laplacian equation. Appl. Math. Lett. 25, 586-589 (2012)

6. Del Pino, M, Manásevich, R, Murúa, A: Existence and multiplicity of solutions with prescribed period for a second order quasilinear ODE. Nonlinear Anal. 18, 79-92 (1992)

7. Fabry, C, Fayyad, D: Periodic solutions of second order differential equations with a $p$-Laplacian and asymmetric nonlinearities. Rend. Ist. Mat. Univ. Trieste 24, 207-227 (1992)

8. Fabry, C, Manásevich, R: Equations with a p-Laplacian and an asymmetric nonlinear term. Discrete Contin. Dyn. Syst. 7, 545-557 (2001)

9. Jiang, M: A Landesman-Lazer type theorem for periodic solutions of the resonant asymmetric $p$-Laplacian equation. Acta Math. Sin. 21, 1219-1228 (2005)

10. Drábek, P: Solvability and Bifurcations of Nonlinear Equations. Pitman Research Notes in Mathematics, vol. 264 (1992)

11. Anane, A, Dakkak, A: Nonexistence of nontrivial solutions for an asymmetric problem with weights. Proyecciones 19, 43-52 (2000)

12. Boccardo, L, Drábek, $P$, Giachetti, D, Kućera, M: Generalization of Fredholm alternative for nonlinear differential operators. Nonlinear Anal. 10, 1083-1103 (1986)

13. Drábek, $\mathrm{P}$, Invernizzi, $\mathrm{S}$ : On the periodic boundary value problem for forced Duffing equation with jumping nonlinearity. Nonlinear Anal. 10, 643-650 (1986)

14. Fonda, A: On the existence of periodic solutions for scalar second order differential equations when only the asymptotic behaviour of the potential is known. Proc. Am. Math. Soc. 119, 439-445 (1993)

15. Habets, P, Omari, P, Zanolin, F: Nonresonance conditions on the potential with respect to the Fučík spectrum for the periodic boundary value problem. Rocky Mt. J. Math. 25, 1305-1340 (1995)

16. Liu, W, Li, Y: Existence of $2 \pi$-periodic solutions for the non-dissipative Duffing equation under asymptotic behaviors of potential function. Z. Angew. Math. Phys. 57, 1-11 (2006)

17. Omari, $\mathrm{P}$, Zanolin, F: Nonresonance conditions on the potential for a second-order periodic boundary value problem. Proc. Am. Math. Soc. 117, 125-135 (1993)

18. Zhang, M: Nonresonance conditions for asymptotically positively homogeneous differential systems: the Fučík spectrum and its generalization. J. Differ. Equ. 145, 332-366 (1998)

19. Zhang, M: The rotation number approach to the periodic Fučík spectrum. J. Differ. Equ. 185, 74-96 (2002)

20. Deimling, K: Nonlinear Functional Analysis. Springer, New York (1985)

doi:10.1186/1687-2770-2013-96

Cite this article as: Chang and Qiao: Existence of periodic solutions for a class of $p$-Laplacian equations. Boundary Value Problems 2013 2013:96.

\section{Submit your manuscript to a SpringerOpen ${ }^{\circ}$ journal and benefit from:}

- Convenient online submission

- Rigorous peer review

- Immediate publication on acceptance

Open access: articles freely available online

- High visibility within the field

- Retaining the copyright to your article 